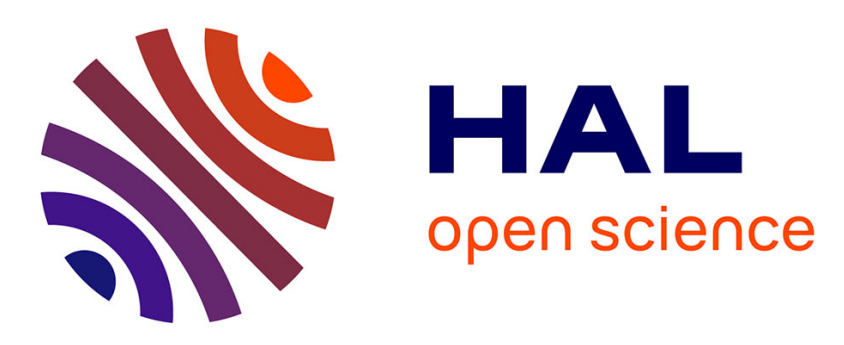

\title{
Semi-Classical Limit and Least Action Principle Revisited with (min,+) Path Integral and Action-Particle Duality
}

Abdelouahab Kenoufi, Michel Gondran, Alexandre Gondran

\section{To cite this version:}

Abdelouahab Kenoufi, Michel Gondran, Alexandre Gondran. Semi-Classical Limit and Least Action Principle Revisited with (min,+) Path Integral and Action-Particle Duality. Russian Journal of Mathematical Physics, 2020, 27 (1), pp.61-75. 10.1134/S1061920820010069 . hal-02892760

\section{HAL Id: hal-02892760 \\ https://hal-enac.archives-ouvertes.fr/hal-02892760}

Submitted on 20 Jul 2020

HAL is a multi-disciplinary open access archive for the deposit and dissemination of scientific research documents, whether they are published or not. The documents may come from teaching and research institutions in France or abroad, or from public or private research centers.
L'archive ouverte pluridisciplinaire HAL, est destinée au dépôt et à la diffusion de documents scientifiques de niveau recherche, publiés ou non, émanant des établissements d'enseignement et de recherche français ou étrangers, des laboratoires publics ou privés. 


\title{
Semi-classical limit and Least Action Principle revisited with $($ min, +$)$ Path Integral and Action-Particle Duality
}

\author{
Abdelouahab KENOUFI ${ }^{1,2}$, Michel GONDRAN ${ }^{2}$ and Alexandre GONDRAN ${ }^{3}$ \\ ${ }^{1}$ Scientific COnsulting for Research \& Engineering (SCORE), Strasbourg, France. \\ kenoufi@s-core.fr \\ ${ }^{2}$ European Interdisciplinary Academy of Sciences (EIAS), Paris, France. \\ michel.gondran@polytechnique.org \\ ${ }^{3}$ Ecole Nationale de l'Aviation Civile (ENAC), Toulouse, France. \\ alexandre.gondran@enac.fr \\ article published in \\ Russian Journal of Mathematical Physics, Vol. 27, No. 1, 2020, pp. 61-75
}

\begin{abstract}
One shows that the Feynman's Path Integral designed for quantum mechanics has an analogous in classical mechanics, the so-called $(\min ,+)$ Path Integral. This former is build on $(\min ,+)$-algebra and $(\min ,+)$-analysis which permit to handle in a linear way non-linear problems occurring in mathematical physics. The Hamilton-Jacobi equations and their solutions within this mathematical framework, are introduced and yield to a new interpretation expressed in a duality between action field and particle.
\end{abstract}

\section{Introduction}

Path integral formalism already exists in classical mechanics[42]. In the 1920's, N. Wiener introduced his real valued functional integral for modeling diffusion and Brownian motions[8]. In 1933, P. A. M. Dirac extended this approach to the use of the Lagrangian in quantum mechanics rather than the Hamiltonian[45]. R.P. Feynman proposed in 1948[15, 12] a complete method to achieve Dirac's program and to retrieve quantum mechanics laws. He showed how the semi-classical limit can be obtained easily within this formalism and that classical mechanics is an approximation of the quantum one[13]. Schrödinger equation can be viewed as a diffusion equation with an imaginary diffusion constant, and the Feynman's Path Integral (FPI) can be thus considered as an analytic continuation of Wiener's Path Integral (WPI). FPI is a well-designed mathematical tool for quantum theory that permits to generalize the least-action principle of classical mechanics[12, 42]. Instead of the classical notion of a single and unique classical trajectory for a system, it uses a functional integral, a sum over histories, meaning a sum over an infinity of quantum-mechanically possible trajectories, in order to compute a quantum probability amplitude. The path integral formalism also relates quantum and stochastic processes, and this has been the starting point and the basis in the 1970s of the unification of Quantum Field Theory and Statistical Field Theory of a fluctuating field near a second-order phase transition.

Tropical and idempotent mathematics find their roots in operations research, theoretical computer 
sciences, automation, and at interface between boolean algebra and real numbers arithmetic. The term tropical has been introduced in honor of the Brazilian mathematician Imre Simon (19432009). The main idea driving those new and original mathematics is to develop algebra and analysis on structures which are not fields or rings, but on dioids or semi-rings, eventually semi-fields. Twenty years before the introduction of (min,+)-analysis by V. P. Maslov et al in 1987 and 1992 $[38,44,16]$ under the name idempotent analysis, some mathematicians and computer scientists have first defined and used ( $\min ,+)$-algebra and $(\max ,+)$-algebra to solve problems in operation research $[9,22,43,40,7]$. This was achieved by replacing usual operations such as + and $\times$ with min or max. Some authors have shown that if ones extends (min, +)-algebra to some similar and comparable algebraic structures such as Peteanu's semi-groups, Benzeken pseudo-lattices, and Carré's semi-rings, those approaches can be applied to the resolution of many path-finding problems in graphs $[25,27,26,29,30,28]$.

In this article, one shows how idempotent mathematics, particularly algebra and analysis in dioids can be the right frameworks to develop powerful and versatile tools for another approach of least action principle in classical mechanics. This permits to reconsider the definition of action according to the initial and final limit conditions and to summarize it in a generalization of the system physical action. One introduces a new type of path integral which proves that this formalism already exists in classical mechanics, and yields to a new interpretation of the duality between particle and action. In the section (2), some interesting idempotent algebraic structures are presented in order to explain that their deformations according to a real parameter can transform a non-linear problem described in a real field, into a linear problem expressed within those deformed frameworks. The example of shortest path research in a graph is exhibited and solved into a particular dioid structure, the so-called $(\mathrm{min},+)$ dioid. It yields naturally to a continuous extension which expresses nothing more than the least action principle in physics. The section (3) introduces some elements of functional analysis in $(\mathrm{min},+)$ dioid, such as integration, scalar product, convolution and Fourier-Legendre transform. In section (4), one reconsiders the derivation of motion equations of a system from the least-action principle according to the initial and final conditions, and the definition of the involved actions, making by this way distinction between Hamilton-Jacobi action, and Euler-Lagrange's one. The section (5) introduces a new kind of path integral, typically expressed within $(\mathrm{min},+)$ dioid and well-suited for describing the least action principle in classical mechanics. It yields to an interpretation of least-action principle in terms of duality between Hamilton-Jacobi action and particle nature. The semi-classical limits of quantum particles description are exhibited and discussed as well. We end in the last section (6) with a conclusion summarizing results and opening perspectives and promising developments.

\section{Elements of idempotent algebra}

Many fields of mathematics have been built and based on groups, rings and fields theories. Those structures try to define inverse of elements for given operations and permit to solve some algebraic equations. Many examples in sciences don't allow such constructions, and one has often to deal with restricted algebraic structures where inversions are not always possible and well-defined. Nevertheless, those "less sophisticated" mathematical frameworks are promising theoretical fields of research since they have a huge potential of modelisation of phenomena occuring in physics, informatics, economics, ... . The first three examples of such algebraic structures are Boolean Algebra for logic, Kleene's Algebra in theoretical informatics for formal languages and automation theory, and $(\mathrm{min},+)$ dioid for shortest path research in a graph. 


\subsection{Dioids, semi-rings, and semi-fields}

The term dioid takes its origin in the work of M. Gondran and was in the book of J. Kutzman about Network Theory [23], in which he described an algebraic structure made from two monoids. Those unconventional structures, semi-rings, semi-fields and dioids [38, 44, 46], come up in many applications such as optimal control, graph theory, classical, quantum and statistical physics for example. They are the basic object of the so-called idempotent mathematics. Let us remind first some algebraic definitions and properties.

Definition 1 - A set $X \neq \emptyset$ endowed with two laws $\oplus$ and $\otimes,(X, \oplus, \otimes)$, is called a semi-ring if the following conditions are verified:

- $\oplus$ and $\otimes$ are associative binary operations,

- $\oplus$ is commutative, and $\otimes$ is distributive with respect to $\oplus$,

- $\exists e_{\otimes} \in X$ such that $e_{\otimes} \otimes x=x \otimes e_{\otimes}=x, \forall x \in X$,

- $\exists e_{\oplus} \in X$ such that $e_{\oplus} \neq e_{\otimes}$ and $e_{\oplus} \oplus x=x$, and $e_{\oplus} \otimes x=x \otimes e_{\oplus}=e_{\oplus}, \forall x \in X$.

Remark 1 Those properties express the fact that $(X, \oplus)$ and $(X, \otimes)$ are respectively commutative monoïds with neutral elements $e_{\oplus}$, and $e_{\otimes}$, besides the distributivity of $\otimes$ according $\oplus$, and the absorbing property of $e_{\oplus}$ for the $\otimes$ operation.

Definition 2 - For a semi-ring $(X, \oplus, \otimes)$, if all elements $x \neq e_{\oplus} \in X$ are invertible for $\otimes$, it is called a semi-field. Moreover, if $x \oplus x=x$ for all $x \in X$ the semi-ring (semi-field) will be qualified as idempotent semi-ring (semi-field).

Definition 3 - The binary relation () defined on monoïd $(X, \oplus)$ by $x \triangleq y \Leftrightarrow \exists z \in X, y=x \oplus z$ is a pre-order relation (reflexive and transitive) called canonical or standard pre-order. If $\ominus$ is an order (anti-symmetric), $(X, \oplus)$ is then called (canonically) ordered monoïd.

Definition 4 - A semi-ring $(X, \oplus, \otimes)$ such that $(X, \oplus)$ is a (canonically) ordered monoïd is called $a$ dioid.

Remark 2 If $\oplus$ is commutative and idempotent, then the pre-order relation $\ominus$ relation is a canonical order.

Example 1 The set of natural numbers $(\mathbb{N},+, \times)$ with neutral elements 0 and 1 , and with $\leq$ as canonical ordered relation, is a dioid, neither a field or a ring.

Example 2 The set of positive real numbers $\left(\mathbb{R}^{+},+, \times\right)$with neutral elements 0 and 1 , and with $\leq$ as canonical ordered relation, is a dioid, a semi-ring and a semi-field.

Example 3 Both dioids $\mathbb{R}_{\max ,+}=(\mathbb{R} \cup\{-\infty\}$, $\max ,+)$ with neutral elements $-\infty$ and $0, \mathbb{R}_{\min ,+}=$ $(\mathbb{R} \cup\{+\infty\}$, min, +$)$ with neutral elements $+\infty$ and 0 , and endowed respectively with order relation $\ominus \equiv \leq$ and $\ominus \equiv \geq$ are idempotent.

Remark 3 If the dioids are $\mathbb{R}_{\min ,+}$ or $\mathbb{R}_{\max ,+}$, and even if the standard order are opposite to the conventional ones, $\min$ and $\max$ have to be understood in the conventional sense:

- In $\mathbb{R}_{\min ,+}, \forall x, y \in \mathbb{R},(x \ominus y) \Leftrightarrow(\exists z \in \mathbb{R}, y=x \oplus z=\min (x, z)) \Longrightarrow(x \geq y)$.

- In $\mathbb{R}_{\max ,+}, \forall x, y \in \mathbb{R},(x \ominus y) \Leftrightarrow(\exists z \in \mathbb{R}, y=x \oplus z=\max (x, z)) \Longrightarrow(x \leq y)$. 


\subsection{Deformation of the dioid $\left(\mathbb{R}^{+},+, \times\right)$}

There are many examples of ordered monoïds, which can be built with mean of algebraic deformation and isomorphism using a parameter $h \in \mathbb{R}^{+*}$ from the monoïds $\left(\mathbb{R}^{+},+\right)$and $\left(\mathbb{R}^{+}, \times\right)$. Thus, one can go further and construct a large number of dioids from the dioid $\left(\mathbb{R}^{+},+, \times\right)$as well $[34,33]$. This former is the natural set for defining and developing measure theory in general and probability theory in particular. In order to achieve that, for any monoïd $\left(X, \oplus_{h}\right) \subset \mathbb{R}$ and any bijection $\Phi_{h}: X \longrightarrow \mathbb{R}^{+}$, one can create an isomorphism between $X$ and $\mathbb{R}^{+}$by transferring the $\oplus_{h}$ operation through $\Phi_{h}$

$$
\Phi_{h}\left(a \oplus_{h} b\right)=\Phi_{h}(a)+\Phi_{h}(b), \forall a, b \in X
$$

which leads to

$$
a \oplus_{h} b=\Phi_{h}^{-1}\left(\Phi_{h}(a)+\Phi_{h}(b)\right) .
$$

The same procedure can be done for the $\otimes_{h}$ operation as well with

$$
a \otimes_{h} b=\Phi_{h}^{-1}\left(\Phi_{h}(a) \times \Phi_{h}(b)\right) .
$$

This endows $\left(X, \oplus_{h}, \otimes_{h}\right)$ with a dioid structure, and particularly one gets for the neutral elements $: e_{\oplus_{h}}=\Phi_{h}^{-1}(0)$ and $e_{\otimes_{h}}=\Phi_{h}^{-1}(1)$.

Remark 4 In classical mechanics, the deformation parameter $h$ corresponds to the diffusion or viscosity constant. It is the Planck constant in quantum physics, and its limit to zero, can be considered as the semi-classical limit. The following example of deformation below is therefore very important for physics applications, especially in statistical and quantum mechanics.

Proposition 1 Let's consider the isomorphism $\Phi_{h}$ from $\left(\mathbb{R}, \oplus_{h}, \otimes_{h}\right)$ to $\left(\mathbb{R}^{+},+, \times\right)$, with paramter $h \in \mathbb{R}^{+*}, \Phi_{h}(x)=e^{-\frac{x}{h}}$ and $\Phi^{-1}(x)=-h \ln (x)$.

Then $\forall a, b \in \mathbb{R}^{+}$,

$$
\left\{\begin{array}{l}
a \oplus_{h} b=-h \ln \left(e^{-\frac{a}{h}}+e^{-\frac{b}{h}}\right), a \otimes_{h} b=a+b, \\
\Phi_{h}^{-1}(0)=+\infty, \Phi_{h}^{-1}(1)=0 .
\end{array}\right.
$$

Since $\otimes_{h}$ is $h$-independent, the deformation of the dioid $\left(X, \oplus_{h}, \otimes_{h}\right)$ for $h \rightarrow 0^{+}$leads to

$$
\lim _{h \rightarrow 0^{+}}\left(a \oplus_{h} b\right)=\min (a, b), \lim _{h \rightarrow 0^{+}}\left(a \otimes_{h} b\right)=a+b .
$$

The structure $\left(\mathbb{R}, \oplus_{h}, \otimes_{h}\right)$ is thus deformed into $\mathbb{R}_{\min ,+}=(\mathbb{R} \cup\{+\infty\}$, min, +$)$ when $h \rightarrow 0^{+}$.

Proposition 2 In the same way, if one changes the bijective mapping to $\Phi_{h}(x)=e^{\frac{x}{h}}$, the deformation of $\left(\mathbb{R}, \oplus_{h}, \otimes_{h}\right)$ gives $\mathbb{R}_{\max ,+}$ when $h \rightarrow 0^{+}$.

Example 4 Let's consider the one-dimensional heat diffusion equation

$$
\frac{\partial u(x, t)}{\partial t}=\frac{h}{2} \frac{\partial^{2} u(x, t)}{\partial x^{2}}
$$

with parameter $h>0$. It is a linear equation since if $u_{1}$ and $u_{2}$ are both solutions of heat equation, then any linear combination $u=\lambda_{1} u_{1}+\lambda_{2} u_{2},\left(\lambda_{1}, \lambda_{2}\right) \in \mathbb{R}^{2}$, is still a solution.

If one makes the following changes $u(x, t)=\Phi_{h}(w(x, t))$, and $u_{i}(x, t)=\Phi_{h}\left(w_{i}(x, t)\right)$, for $i \in\{1,2\}$, the equation (4) becomes a non-linear one called Bürgers equation 


$$
\frac{\partial w(x, t)}{\partial t}+\frac{1}{2}\left(\frac{\partial w(x, t)}{\partial x}\right)^{2}-\frac{h}{2} \frac{\partial^{2} w(x, t)}{\partial x^{2}}=0,
$$

and then $w=\left(\lambda_{1} \otimes_{h} u_{1}\right) \oplus_{h}\left(\lambda_{2} \otimes_{h} u_{2}\right)=-h \ln \left(e^{-\frac{w_{1}+\lambda_{1}}{h}}+e^{-\frac{w_{2}+\lambda_{2}}{h}}\right)$ is still a solution. One deduces immediately that Bürgers equation is linear in the doid defined by relations (3).

At the limit $h \rightarrow 0$, equation (5) becomes a one-dimensional Hamilton-Jacobi equation, and $w=$ $\min \left\{\lambda_{1}+u_{1}, \lambda_{2}+u_{2}\right\}[38,34,33]$.

\subsection{The shortest path problem in graph theory and Bellman's Optimal- ity Theorem}

In Operation Research, graphs can be used to represent paths, flow circulations, and pre-order relations for instance. Almost all arcs or edges are valued, which means that they are endowed with a real number which might represent physical actions, distances, time intervals, or flow capacities, etc.

Several interesting problems occurring in this field consist to find an path in a graph with an optimal valuation (sum of valuations). Most algorithms designed to solve such kind of problems are based on the Bellman's Optimality Theorem, which is the keystone of Dynamic Programming Theory $[2,34,33]$. It states that for a given criterium in a graph, every part of an optimal path, is optimal for the same criterium. Its proof is obvious with mean of contradiction reasoning : if the sub-path is not optimal, one can replace it with a better one, and then the path from which it is extracted is not optimal, which is contrary to the initial hypothesis. (min, + )analysis takes it roots from the shortest path research in a finite graph [18, 19, 34, 33]. Carré and M. Gondran et al [34,33] have shown that the optimality equation to determine the shortest path is a linear equation with fixed-point solution in a particular algebraic structure : the dioid $\mathbb{R}_{\text {min, }+}=(\mathbb{R} \cup\{+\infty\}$, min, +$)$ which is an idempotent semi-ring different from real numbers field $(\mathbb{R},+, \times)[44,38,34,33]$. They have demonstrated that the classical resolution methods of linear algebra on the real numbers field can be re-written into this dioid $\mathbb{R}_{\text {min }}$ yielding to computation algorithms in order to find the shortest path. In the same spirit, if one uses the dioid $\mathbb{R}_{\max , \min }=(\mathbb{R} \cup\{+\infty\}, \max , \min )$, it is possible to solve other problems such as to find maximal capacity path in a graph. Almost all usual concepts used in analysis for $\mathbb{R}$ can be transfered and studied in dioids, in particular in $\mathbb{R}_{\min ,+}$, such as eigenvectors and eigenvalues calculations, linear dependence, determinants computations.

Let's consider a directed graph $G=(X, U)$ with $n \in \mathbb{N}^{*}$, where $X=\left\{X_{i}, i \in \llbracket 0, n \rrbracket\right\}$ and $U=\left\{\left(X_{i}, X_{j}\right) \in X^{2},(i, j) \in \llbracket 0, n \rrbracket^{2}\right\}$ are respectively the sets of numbered vertices and arcs. A real number $a_{i j}$ is assigned to each arc $\left(X_{i}, X_{j}\right) \in U$, as the length between vertices $X_{i}$ and $X_{j}$.

Remark 5 Without lost of generalization and for sake of simplicity, in this article, one considers a 1-graph $G$, which means that for any pair of vertices $\left(X_{i}, X_{j}\right) \in X^{2}$, it exists[18] at most one arc of the form $\left(X_{i}, X_{j}\right) \in U$. Moreover, one supposes they are no circuits with negative lengths appearing in the graph $G$.

One defines $i_{0}=0$ as the origin vertex index, and we seek the shortest path length $Y_{j}$ between the vertices $X_{i_{0}}=X_{0}$ and the other vertices $\left\{X_{j}\right\}_{j \in \llbracket 1, n \rrbracket}$ of the graph. The Dynamic Programming Bellman's theorem yields for $Y_{j}$ to the following relations $[2,43,34,33]$

$$
\forall j \in \llbracket 1, n \rrbracket, Y_{j}=\min _{X_{i} \in \Gamma_{j}^{-1}}\left\{Y_{i}+a_{i j}\right\}, \text { with } Y_{0}=0,
$$




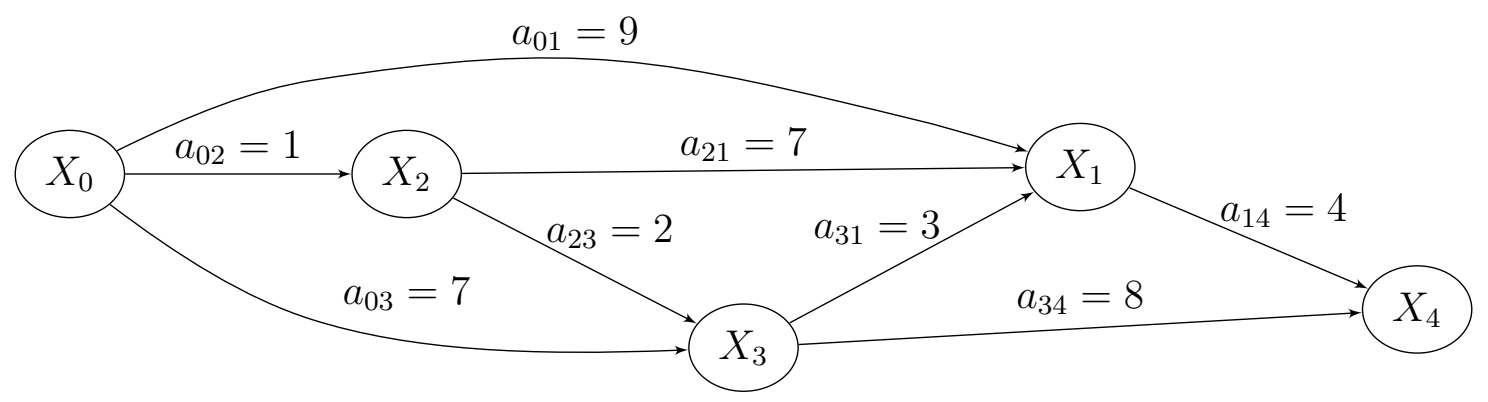

Figure 1: Example of shortest path problem in a 5-order directed graph with positive lengths and without circuits. The minimal length from $X_{0}$ to $X_{4}$ is $Y_{4}=a_{02}+a_{23}+a_{31}+a_{14}=1+2+3+4=10$ and corresponds to the path $\left(X_{0}, X_{2}, X_{3}, X_{1}, X_{4}\right)$.

where $\Gamma_{j}^{-1}=\left\{X_{i},\left(X_{i}, X_{j}\right) \in U\right\}$ is the set of direct predecessors of vertex $X_{j}$ in G. If one sets the length of missing arcs to $+\infty$, the previous equations can be rewritten as

$$
\forall j \in \llbracket 1, n \rrbracket, Y_{j}=\min _{i \in \llbracket 0, n \rrbracket}\left\{Y_{i}+a_{i j}\right\}, \text { with } Y_{0}=0,
$$

which is equivalent to

$$
\forall j \in \llbracket 1, n \rrbracket, Y_{j}=\min \left\{\min _{i \in \llbracket 1, n \rrbracket}\left\{Y_{i}+a_{i j}\right\}, a_{0 j}\right\}, \text { with } Y_{0}=0 .
$$

Let us consider the dioid $\left(\overline{\mathbb{R}}^{+}, \oplus, \otimes\right) \equiv\left(\mathbb{R}^{+} \cup\{+\infty\}\right.$, min, +$)$, the former equations can be written then in a linear way

$$
\forall j \in \llbracket 1, n \rrbracket, Y_{j}=\left(\bigoplus_{i=1}^{n} Y_{i} \otimes a_{i j}\right) \oplus\left(a_{0 j}\right) .
$$

Let $\mathbf{A}=\left(a_{i j}\right)^{\top}$ the transpose of the lengths matrix, with $(i, j) \in \llbracket 1, n \rrbracket^{2}, \mathbf{B}=\left(a_{0 j}\right)$, and $\mathbf{Y}=\left(Y_{j}\right)$, $j \in \llbracket 1, n \rrbracket$. Then the equation (6) can be written as a fixed point one

$$
\mathbf{Y}=\mathbf{A} \otimes \mathbf{Y} \oplus \mathbf{B} .
$$

Seeking for the shortest path from the origin vertex to others consists to solve the linear system (7) in the algebraic structure $\mathbb{R}_{\min ,+}$.

Remark 6 Even if a dioid in general, and $\mathbb{R}_{\min ,+}$ in particular is an algebraic structure with restricted properties compared to others such as rings, fields, or linear vector spaces, one can define and adapt most resolution algorithms of linear systems in a field (Jacobi, Gauss-Seidel, Gauss methods, ...), and to develop some schemes specific to dioids $\mathbb{R}_{\min ,+}[1$, 34,33]. For example, the resolution of equations (7) can be performed with mean of the nilpotent property of matrix $\mathbf{A}$, and with a generalization and extension of the well-known Taylor serie development $(1-\mathbf{A})^{-1}=\sum_{k \geq 0} \mathbf{A}^{k}$ to the dioid $\mathbb{R}_{\min ,+}$.

Example 5 Considering the simple and pedagogical example exhibited in Figure (1), the previous equation (7) gives

$$
\left(\begin{array}{l}
Y_{1} \\
Y_{2} \\
Y_{3} \\
Y_{4}
\end{array}\right)=\left(\begin{array}{cccc}
0 & +7 & 3 & +\infty \\
+\infty & 0 & +\infty & +\infty \\
+\infty & 2 & 0 & +\infty \\
+4 & +\infty & +8 & 0
\end{array}\right) \otimes\left(\begin{array}{c}
Y_{1} \\
Y_{2} \\
Y_{3} \\
Y_{4}
\end{array}\right) \oplus\left(\begin{array}{c}
9 \\
1 \\
7 \\
+\infty
\end{array}\right)=\left(\begin{array}{c}
\min \left\{Y_{1}, 7+Y_{2}, 3+Y_{3}, 9\right\} \\
\min \left\{Y_{2}, 1\right\} \\
\min \left\{2+Y_{2}, Y_{3}, 7\right\} \\
\min \left\{4+Y_{1}, 8+Y_{3}, Y_{4}\right\}
\end{array}\right)
$$


The Jacobi algorithm gives the exact result and consists in a finite number of iterations

$$
\mathbf{Y}^{(k+1)}=\mathbf{A} \otimes \mathbf{Y}^{(k)} \oplus \mathbf{B}, \forall k \in \llbracket 1,4 \rrbracket, \text { with }\left(\mathbf{Y}^{(0)}\right)_{j}=+\infty, \forall j \in \llbracket 1,4 \rrbracket .
$$

The solution of (8) is then given as $\mathbf{Y}=\mathbf{Y}^{(4)}$ as shown below

$$
\mathbf{Y}^{(0)}=\left(\begin{array}{c}
+\infty \\
+\infty \\
+\infty \\
+\infty
\end{array}\right) \Longrightarrow \mathbf{Y}^{(1)}=\left(\begin{array}{c}
9 \\
1 \\
7 \\
+\infty
\end{array}\right) \Longrightarrow \mathbf{Y}^{(2)}=\left(\begin{array}{c}
8 \\
1 \\
3 \\
13
\end{array}\right) \Longrightarrow \mathbf{Y}^{(3)}=\left(\begin{array}{c}
6 \\
1 \\
3 \\
11
\end{array}\right) \Longrightarrow \mathbf{Y}^{(4)}=\left(\begin{array}{c}
6 \\
1 \\
3 \\
10
\end{array}\right)
$$

\section{Elements of idempotent analysis}

The starting point of idempotent analysis is based on the works of V. P. Maslov et al [38, 44, 46], and many other authors [39, 18]. Maslov introduced (min, +)-analysis in the Chapter (8) of his book about Linear Equations Theory in the semi-modulus and called it idempotent analysis [38]. Some mathematical objects such as integration, scalar product and Fourier-Legendre transform have their analogous in idempotent analysis. One presents them below in the case of $(\mathrm{min},+)$-analysis .

\subsection{Integration}

Let's define $X=(\mathbb{R},+, \times)$ and $Y=\mathbb{R}_{\min ,+}$. One considers the function $f: X \longrightarrow Y$, and the $Y$-semi-module $\mathcal{B}(X, Y)$ of all functions $X \longrightarrow Y$ that are bounded in the sense of the standard order on $Y$. The idempotent analog of a linear functional space is a set of $Y$-valued functions that is closed under addition and multiplication of functions by elements of $Y$, or an $Y$-semi-module. From a heuristic point of view, a $\oplus$-Riemann sum of the form $\sum_{i} f\left(x_{i}\right) \cdot \Delta x_{i}$ corresponds to the expression $\bigoplus_{i} f\left(x_{i}\right) \otimes \Delta x_{i}=\min _{i}\left\{f\left(x_{i}\right)+\Delta x_{i}\right\}$.

Definition 5 - If $Y=\mathbb{R}_{\min ,+}$ or $Y=\mathbb{R}_{\max ,+}$, and $\forall \varphi \in \mathcal{B}(X, Y)$, the idempotent analog of integration is then respectively defined by

$$
\int_{X}^{\oplus} f(x) d x \equiv \min _{x \in X} f(x), \text { or } \int_{X}^{\oplus} f(x) d x \equiv \max _{x \in X} f(x) .
$$

\subsection{Scalar product}

One can replace the classical scalar product $\langle f, g\rangle=\int_{x \in X} f(x) \cdot g(x) \cdot d x$ with the $($ min, +$)$ scalar product $[18,32]$

$$
\langle f, g\rangle_{(\min ,+)} \equiv \int_{x \in X}^{\oplus} f(x) \otimes g(x) \otimes d x=\min _{x \in X}\{f(x)+g(x)\} .
$$

Proof 1 One reminds below the demonstration that it is a scalar product within the $(\mathrm{min},+)$ dioid is straightforward [32].

- Symmetry : Obviously, $\langle f, g\rangle_{(\min ,+)}=\langle g, f\rangle_{(\min ,+)}$. 


\section{- Positive-definiteness}

Since $+\infty$ is the neutral element of the min operator, if $\langle f, f\rangle_{(\min ,+)}=+\infty$, then $f(x)=+\infty$ for all $x \in X$. Furthermore, since $\ominus$ in the dioid $\mathbb{R}_{\min ,+}$ corresponds to $\leq$ in the field of real numbers, and all functions are bounded by $+\infty$, one has $\langle f, f\rangle_{(\min ,+)} \ominus+\infty$.

\section{- Bi-linearity}

One has to show that $\langle f, g\rangle_{(\min ,+)}$ is distributive according to $\min$, which means $\left\langle f, \min \left\{g_{1}, g_{2}\right)\right\rangle_{(\min ,+)}=$ $\min \left\{\left\langle f, g_{1}\right\rangle_{(\min ,+)},\left\langle f, g_{2}\right\rangle_{(\min ,+)}\right\}$, and linear according to the addition of a scalar $\lambda:\langle f(x), \lambda+$ $g(x)\rangle_{(\min ,+)}=\lambda+\langle f, g\rangle_{(\min ,+)}$. The linearity is obvious since $\min _{x \in X}\{f(x)+\lambda+g(x)\}=$ $\lambda+\min _{x \in X}\{f(x)+g(x)\}$. Distributivity is obtained in two steps. One has first to prove this equality with mean of two inequalities. We start first with the simple relations

$$
\left\langle f, g_{1}\right\rangle_{(\min ,+)} \leqslant f(x)+g_{1}(x), \text { and }\left\langle f, g_{2}\right\rangle_{(\min ,+)} \leqslant f(x)+g_{2}(x), \forall x .
$$

This gives $\min \left\{\left\langle f, g_{1}\right\rangle_{(\min ,+)},\left\langle f, g_{2}\right\rangle_{(\min ,+)}\right\} \leqslant \min \left\{f(x)+g_{1}(x), f(x)+g_{2}(x)\right\} \forall x$.

And since

$$
\min \left\{f(x)+g_{1}(x), f(x)+g_{2}(x)\right\}=f(x)+\min \left\{g_{1}(x), g_{2}(x)\right\},
$$

one has $\min \left\{\left\langle f, g_{1}\right\rangle_{(\min ,+)},\left\langle f, g_{2}\right\rangle_{(\min ,+)}\right\} \leqslant f(x)+\min \left\{g_{1}(x), g_{2}(x)\right\} \forall x$, which yields to the inequality

$$
\min \left\{\left\langle f, g_{1}\right\rangle_{(\min ,+)},\left\langle f, g_{2}\right\rangle_{(\min ,+)}\right\} \leqslant\left\langle f, \min \left\{g_{1}, g_{2}\right\}\right\rangle_{(\min ,+)} .
$$

In a second step, one can write

$$
\left\langle f, \min \left\{g_{1}, g_{2}\right\}\right\rangle_{(\min ,+)} \leqslant f(x)+\min \left\{g_{1}(x), g_{2}(x)\right\} \leqslant f(x)+g_{1}(x) \forall x,
$$

which becomes

$$
\left\langle f, \min \left\{g_{1}, g_{2}\right\}\right\rangle_{(\min ,+)} \leqslant\left\langle f, g_{1}\right\rangle_{(\min ,+)} .
$$

and in the same manner

$$
\left\langle f, \min \left\{g_{1}, g_{2}\right\}\right\rangle_{(\min ,+)} \leqslant f(x)+\min \left\{g_{1}(x), g_{2}(x)\right\} \leqslant f(x)+g_{2}(x) \forall x,
$$

giving now

$$
\left\langle f, \min \left\{g_{1}, g_{2}\right\}\right\rangle_{(\min ,+)} \leqslant\left\langle f, g_{2}\right\rangle_{(\min ,+)},
$$

and then from (11) and (12)

$$
\left\langle f, \min \left\{g_{1}, g_{2}\right\}\right\rangle_{(\min ,+)} \leqslant \min \left\{\left\langle f, g_{1}\right\rangle_{(\min ,+)},\left\langle f, g_{2}\right\rangle_{(\min ,+)}\right\} .
$$

From relations (10) and (13), one deduces finally the equality and thus the distributivity.

One obtains a distribution-like theory : the $(\mathrm{min},+)$ scalar product is linear and continuous in the dioid structure $\mathbb{R}_{\min ,+}=(\mathbb{R} \cup\{+\infty\}$, min, +$)$, non-linear and continuous in the classical structure $(\mathbb{R},+, \times)$. The non-linear distribution $\delta_{(\min ,+)}$ defined on $\mathbb{R}^{n}$ as $\delta_{(\min ,+)}(\mathbf{x})=\{0$ if $\mathbf{x}=\mathbf{0},+\infty$ else $\}$ is analogous in $(\mathrm{min},+)$ analysis to the classical Dirac distribution. Thus, one has

$$
\begin{aligned}
\left\langle\delta_{(\min ,+)}, f\right\rangle_{(\min ,+)} & =\min _{\mathbf{x} \in X}\left\{\delta_{(\min ,+)}(\mathbf{x})+f(\mathbf{x})\right\} \\
& =\min \{f(\mathbf{0}),+\infty\}=f(\mathbf{0}) .
\end{aligned}
$$

It is therefore interesting to study analog results developed in Hilbert spaces functional analysis such as Riesz theorems, Fourier transforms, spectral analysis, and measure theory for example [44, $34,33]$. 


\subsection{Convolution and Fourier-Legendre transform}

Let $(X, \diamond)$ be a group, and $\mathcal{B}\left(X, \mathbb{R}_{\min ,+}\right)$ the set of all bounded functions from $X$ to $\mathbb{R}_{\min ,+}$.

Definition 6 - The convolution product $\circledast$ is defined as, $\forall(x, y) \in X^{2}, \forall(\varphi, \psi) \in \mathcal{B}^{2}\left(X, \mathbb{R}_{\min ,+}\right)$

$$
(\varphi \circledast \psi)(x)=\int_{X}^{\oplus} \varphi(y) \otimes \psi\left(y^{-1} \diamond x\right) \otimes d y=\min _{y \in X}\left\{\varphi(y)+\psi\left(y^{-1} \diamond x\right)\right\},
$$

Remark $7 \mathcal{B}\left(X, \mathbb{R}_{\min ,+}\right)$ is an idempotent semi-ring with respect to the following analog $\circledast$ of the usual convolution.

Let $X=\mathbb{R}^{n}$, considered as a topological group with respect to the vector addition. The conventional Fourier transform $\tilde{\varphi}$ of $\varphi$ is defined as

$$
\tilde{\varphi}(\xi)=\int_{X} e^{-i \xi \cdot \mathbf{x}} \varphi(\xi) d \mathbf{x}
$$

where $e^{i \xi \cdot \mathbf{x}}$ is a character of the group $X$, i.e., a solution of the following functional equation

$$
\varphi(\mathbf{x}+\mathbf{y})=\varphi(\mathbf{x}) \cdot \varphi(\mathbf{y}) .
$$

The idempotent analog of this equation becomes then

$$
\varphi(\mathbf{x}+\mathbf{y})=\varphi(\mathbf{x}) \otimes \varphi(\mathbf{y})=\varphi(\mathbf{x})+\varphi(\mathbf{y}) .
$$

The continuous idempotent characters are linear functions of the form $\mathbf{x} \longmapsto \xi \cdot \mathbf{x}$ with $\xi \in X$ and this yields to the definition given below.

Definition 7 - The analog of Fourier transform on $\mathcal{B}\left(\mathbb{R}^{n}, \mathbb{R}_{\min ,+}\right)$ can be defined as

$$
\varphi(\mathbf{x}) \longmapsto \tilde{\varphi}(\xi)=\int_{X}^{\oplus}-(\xi \cdot \mathbf{x}) \otimes \varphi(\mathbf{x}) \otimes d \mathbf{x}=\min _{\mathbf{x} \in G}\{\varphi(\mathbf{x})-\xi \cdot \mathbf{x}\}
$$

Remark 8 This transform on $\mathcal{B}\left(\mathbb{R}^{n}, \mathbb{R}_{\min ,+}\right)$ is nothing but the Legendre transform [38], which establish for instance the correspondence between the Lagrangian and the Hamiltonian formulations in classical mechanics.

\section{Euler-Lagrange and Hamilton-Jacobi actions}

When one tries to find the minimal path in a continuous space, optimality equations given by the classical variational calculus are the so-called (deterministic) Hamilton-Jacobi equations which link the time derivative of the action $S(\mathbf{x}, t)$ to the Hamiltonian of the system $H(\mathbf{x}, \nabla S ; t) \equiv$ $\frac{(\nabla S(\mathbf{x}, t))^{2}}{2 m}+V(\mathbf{x})$, and express mathematically the Least Action Principle (LAP)

$$
\left\{\begin{array}{l}
\frac{\partial S(\mathbf{x}, t)}{\partial t}+H(\mathbf{x}, \nabla S ; t)=0 \\
S\left(\mathbf{x}, t_{0}\right)=S_{0}(\mathbf{x})
\end{array}\right.
$$


In the case where presence probability density is defined for the particle, one has to had the continuity equations linking probability density to the action, giving thus the so-called (statistical) Hamilton-Jacobi equations

$$
\left\{\begin{array}{l}
\frac{\partial}{\partial t} S(\mathbf{x}, t)+H(\mathbf{x}, \nabla S ; t)=0 \\
S\left(\mathbf{x}, t_{0}\right)=S_{0}(\mathbf{x}), \\
\frac{\partial}{\partial t} \rho(\mathbf{x}, t)+\nabla \cdot\left(\rho(\mathbf{x}, t) \cdot \frac{\nabla S(\mathbf{x}, t)}{m}\right)=0, \\
\rho\left(\mathbf{x}, t_{0}\right)=\rho_{0}(\mathbf{x}) .
\end{array}\right.
$$

One reminds that the velocity field is given by $\mathbf{v}(\mathbf{x}, t)=\frac{\nabla S(\mathbf{x}, t)}{m}$ for particle with mass $m$.

Remark 9 Equations (15) are non-linear on the real numbers field $(\mathbb{R},+, \times)$. Maslov et al [38, 44] have shown that this equation is linear in the dioid $\mathbb{R}_{\min }=(\mathbb{R} \cup\{+\infty\}$, min, +$)$ : thus, if $S_{1}(\mathbf{x}, t)$ et $S_{2}(\mathbf{x}, t)$ are solutions of Hamilton-Jacobi equations $(15,16)$, then $\min _{\mathbf{x}}\left\{\lambda+S_{1}(\mathbf{x}, t), \mu+S_{2}(\mathbf{x}, t)\right\}$ for all $\lambda, \mu \in \mathbb{R}$, is a solution of the same equations $(15,16)$.

Definition 8 (Classical discerned particle) - A classical particle is said to be discerned prepared, if one knows, at the initial time its position $\mathbf{x}_{0}$ and its velocity $\mathbf{v}_{0}$.

Definition 9 (Classical undiscerned particle) - A classical particle is said to be undiscerned when only its initial probability density $\rho_{0}(\mathbf{x})$ and its initial action $S_{0}(\mathbf{x})$ are defined.

Remark 10 For an undiscerned classical massive particle $(m)$, with initial probability density of presence $\rho_{0}(\mathbf{x})$ and initial action $S_{0}(\mathbf{x})$, the probability density $\rho(\mathbf{x}, t)$ and the action $S(\mathbf{x}, t)$ verify the (statistical) Hamilton-Jacobi equations.

Definition 10 - For the given events $\left(\mathbf{x}_{0}, t_{0}\right)$ and $(\mathbf{x}, t)$, the Euler-Lagrange action $S_{E L}\left(\mathbf{x}, t ; \mathbf{x}_{0}, t_{0}\right)$ is the functional defined by

$$
S_{E L}\left(\mathbf{x}, t ; \mathbf{x}_{0}, t_{0}\right)=\min _{\mathbf{u}(s), s \in\left[t_{0}, t\right]}\left\{\int_{t_{0}}^{t} L(\mathbf{r}(s), \mathbf{u}(s) ; s) d s\right\},
$$

where the minimum (or more generally an extremum) is taken on the velocity $\mathbf{u}(s)$ which is the control variable, for $s \in\left[t_{0}, t\right]$, with the state $\mathbf{r}(s)$ given by the equations

$$
\left\{\begin{array}{l}
\frac{d}{d s} \mathbf{r}(s)=\mathbf{u}(s) \text { for } s \in\left[t_{0}, t\right] \\
\mathbf{r}\left(t_{0}\right)=\mathbf{x}_{0}, \mathbf{r}(t)=\mathbf{x}
\end{array}\right.
$$

This is the Least Action Principle defined by Euler [11] in 1744 and Lagrange [24] in 1755.

If the Lagrangian $L$ is differentiable according to the position $\mathbf{r}$, and $L(\mathbf{r}(s), \dot{\mathbf{r}}(s) ; s)=\frac{1}{2} m \dot{\mathbf{r}}^{2}(s)-$ $V(\mathbf{r})$, the solutions of (17) satisfy the Euler-Lagrange equations on the interval $\left[t_{0}, t\right]$

$$
\left\{\begin{array}{l}
\left(\frac{d}{d s} \frac{\partial}{\partial \dot{\mathbf{r}}}-\frac{\partial}{\partial \mathbf{r}}\right) L(\mathbf{r}(s), \dot{\mathbf{r}}(s) ; s)=0 \quad \text { for } s \in\left[t_{0}, t\right] \\
\mathbf{r}\left(t_{0}\right)=\mathbf{x}_{0}, \mathbf{r}(t)=\mathbf{x}
\end{array}\right.
$$

Let's show how $(\mathrm{min},+)$-analysis can help to explain the differences between Hamilton-Jacobi and Euler-Lagrange actions. 
Remark 11 The Euler-Lagrange action $S_{E L}$ is the elementary solution of the Hamilton-Jacobi equations (15) in (min, +)-analysis with the initial condition

$$
S_{0}(\mathbf{r}) \equiv S\left(\mathbf{r}, t_{0}\right)=\delta_{(\min ,+)}\left(\mathbf{r}-\mathbf{x}_{0}\right)= \begin{cases}0 & \text { if } \mathbf{r}=\mathbf{x}_{0} \\ +\infty & \text { otherwise }\end{cases}
$$

Theorem 1 The (deterministic) Hamilton-Jacobi Action defined as $S_{H J}(\mathbf{x}, t)=\min _{\mathbf{x}_{0}}\left\{S_{0}\left(\mathbf{x}_{0}\right)+\right.$ $\left.S_{E L}\left(\mathbf{x}, t ; \mathbf{x}_{0}, t_{0}\right)\right\}$, is solution of (deterministic) Hamilton-Jacobi equations (15).

Proof 1 According to the Bellman's Theorem[2], Hamilton-Jacobi action $S_{H J}(\mathbf{x}, t)$ verifies the optimality equation on the time interval $[t-\Delta t, t]$ :

$$
S_{H J}(\mathbf{x}, t)=\min _{\substack{\mathbf{u}(s) \\ t-\Delta t \leq s \leq t}}\left\{S_{H J}\left(\mathbf{x}-\int_{t-\Delta t}^{t} \mathbf{u}(s) d s, t\right)+\int_{t-\Delta t}^{t} L\left(\mathbf{x}-\int_{s}^{t} \mathbf{u}(w) d w, \mathbf{u}(s) ; s\right) d s\right\} .
$$

If one supposes that $S$ is differentiable in $\mathbf{x}$ and $t, L$ in $\mathbf{x}, \boldsymbol{u}, t$ and $\boldsymbol{u}$ continuous in time, then previous equation yields to

$$
S_{H J}(\mathbf{x}, t)=\min _{\mathbf{u}(t)}\left\{S_{H J}(\mathbf{x}, t)-\nabla S_{H J}(\mathbf{x}, t) \cdot \frac{d \mathbf{x}(t)}{d t} \Delta t-\frac{\partial S_{H J}(\mathbf{x}, t)}{\partial \mathbf{t}} \Delta t+L((\mathbf{x}, t), t) \Delta t+\circ(\Delta t)\right\},
$$

where one uses the notation $\frac{\partial}{\partial \mathbf{x}} \equiv \nabla$.

Cancelling $S_{H J}(\mathbf{x}, t)$ on both sides and dividing by $\Delta t$, one obtains this important relation when $\Delta t \rightarrow 0^{+}$

$$
\frac{\partial S_{H J}(\mathbf{x}, t)}{\partial t}=\min _{\mathbf{u}}\left\{L(\mathbf{x}, \mathbf{u}, t)-\mathbf{u} \cdot \nabla S_{H J}(\mathbf{x}, t)\right\}
$$

For the Lagrangian $L(\mathbf{x}, \mathbf{u} ; s)=\frac{1}{2} m \mathbf{u}^{2}(s)-V(\mathbf{x}, s)$, the right-hand-side term is the opposite of its Legendre-transform, which is the Hamiltonian $H(\mathbf{x}, \nabla S ; t)=\frac{(\nabla S(\mathbf{x}, t))^{2}}{2 m}+V(\mathbf{x})$. Finally equation (21) yields to a Hamilton-Jacobi-like equation for $S_{H J}$

$$
\frac{\partial S_{H J}(\mathbf{x}, t)}{\partial t}+H\left(\mathbf{x}, \nabla S_{H J} ; t\right)=0
$$

Remark 12 The Hamilton-Jacobi and Euler-Lagrange actions, respectively $S_{H J}$ and $S_{E L}$ are both solutions of Partial Differential Equations (PDE), the Hamilton-Jacobi ones. Moreover, $S_{E L}$ verifies Ordinary Differential Equations (ODE), the so-called Euler-Lagrange equations. Let's remind that those two actions are solutions of problems with different boundary conditions :

- The Euler-Lagrange (or classical) action $S_{E L}\left(\mathbf{x}, t ; \mathbf{x}_{0}, t_{0}\right)$ links the initial position $\mathbf{x}_{0}$ at time $t_{0}$ to the position $\mathbf{x}$ at time $t$;

- The Hamilton-Jacobi action $S_{H J}$ links the initial action $S_{0}$ to the position $\mathbf{x}$ at time $t$;

- While the Euler-Lagrange case entails an unknown initial velocity, the Hamilton-Jacobi one implies an unknown initial position.

Example 6 For a particle in a linear potential $V(\mathbf{x})=-\boldsymbol{K} \cdot \mathbf{x}, \boldsymbol{K} \in \mathbb{R}^{3}$, with initial Hamilton-Jacobi action $S_{0}(\mathbf{x})=m \mathbf{v}_{0} \cdot \mathbf{x}$, one deduces from (22) the Hamilton-Jacobi action:

$$
S_{H J}(\mathbf{x}, t)=m \mathbf{v}_{0} \cdot \mathbf{x}-\left(\frac{1}{2} m \mathbf{v}_{0}^{2}-\boldsymbol{K} \cdot \mathbf{x}\right) t-\frac{1}{2}\left(\boldsymbol{K} \cdot \mathbf{v}_{0}\right) t^{2}-\frac{\boldsymbol{K}^{2}}{6 m} t^{3} .
$$




\section{5 (min, +)-Path Integral and semi-classical limit}

\section{1 (min, +$)$ Action Path Integral}

One can write Hamilton-Jacobi action in a more elegant manner as follow

$$
\begin{aligned}
S_{H J}(\mathbf{x}, t) & =\min _{\mathbf{x}_{0}}\left\{S_{0}\left(\mathbf{x}_{0}\right)+S_{E L}\left(\mathbf{x}, t ; \mathbf{x}_{0}, t_{0}\right)\right\} \\
& =\int^{\oplus} d \mathbf{x}_{0} \otimes S_{E L}\left(\mathbf{x}, t ; \mathbf{x}_{0}, t_{0}\right) \otimes S_{0}\left(\mathbf{x}_{0}\right) .
\end{aligned}
$$

The analogy between (23) and the Schrödinger's wave function $\psi$ with mean of the propagator $G$ and Feynman's Path Integral[13] is straightforward since

$$
\begin{aligned}
\psi(\mathbf{x}, t) & =\int d \mathbf{x}_{0} \cdot G\left(\mathbf{x}, t ; \mathbf{x}_{0}, t_{0}\right) \cdot \psi_{0}\left(\mathbf{x}_{0}\right) \\
& =\int d \mathbf{x}_{0} \cdot \psi_{0}\left(\mathbf{x}_{0}\right) \cdot \int_{\mathbf{x}_{0}, t_{0}}^{\mathbf{x}, t} \mathcal{D}[\mathbf{y}] \cdot e^{\frac{i}{\hbar} S_{E L}\left(\mathbf{y}, t \mid \mathbf{x}_{0}, t_{0}\right)}, \text { with } \psi_{0}\left(\mathbf{x}_{0}\right)=\psi\left(\mathbf{x}_{0}, t_{0}\right) .
\end{aligned}
$$

It is easy to check that $S_{E L}$ verifies a Chapman-Kolmogorov-like formula[12, 42]

Theorem 2 For given space-time points $\left(\mathbf{x}_{0}, t_{0}\right),\left(\mathbf{x}^{\prime}, t^{\prime}\right)$ and $(\mathbf{x}, t)$ with $t_{0}<t^{\prime}<t$, one has the following relation

$$
S_{E L}\left(\mathbf{x}, t ; \mathbf{x}_{0}, t_{0}\right)=\int^{\oplus} d \mathbf{x}^{\prime} \otimes S_{E L}\left(\mathbf{x}, t ; \mathbf{x}^{\prime}, t^{\prime}\right) \otimes S_{E L}\left(\mathbf{x}^{\prime}, t^{\prime} ; \mathbf{x}_{0}, t_{0}\right)
$$

Proof 2 With mean of the Feynman-Dirac formula[12] and $\forall \tau \in\left[t_{0}, t\right]$,

$$
\begin{aligned}
S_{E L}\left(\mathbf{x}, t ; \mathbf{x}_{0}, t_{0}\right) & =\min _{\mathbf{u}(s), s \in\left[t_{0}, t\right]}\left\{\int_{t_{0}}^{t} L(\mathbf{r}(s), \mathbf{u}(s) ; s) d s\right\} \\
& =\min _{\mathbf{u}(s), s \in\left[t_{0}, t\right]}\left\{\int_{t_{0}}^{\tau} L(\mathbf{r}(s), \mathbf{u}(s) ; s) d s+\int_{\tau}^{t} L(\mathbf{r}(s), \mathbf{u}(s) ; s) d s\right\} \\
& =\min _{\mathbf{r}(\tau), \tau \in\left[t_{0}, t\right]}\left\{S_{E L}\left(\mathbf{r}(\tau), \tau ; \mathbf{x}_{0}, t_{0}\right)+S_{E L}(\mathbf{x}, t ; \mathbf{r}(\tau), \tau)\right\} \\
& =\int_{\mathbf{r}(\tau), \tau \in\left[t_{0}, t\right]}^{\oplus} d \mathbf{r} S_{E L}\left(\mathbf{r}(\tau), \tau ; \mathbf{x}_{0}, t_{0}\right) \otimes S_{E L}(\mathbf{x}, t ; \mathbf{r}(\tau), \tau),
\end{aligned}
$$

and one states that $S_{E L}$ is the propagator of the action for the Hamilton-Jacobi equations (15) in (min, +)-algebra . The Euler-Lagrange action $S_{E L}$ can be potentially written as functional integral within this algebra, namely a $(\min ,+)$-path integral.

Definition 11 For time interval $\left[t_{0}, t\right]$ divided into $N$ equal parts, $N \in \mathbb{N}^{*}$, with length $\Delta t=\frac{t-t_{0}}{N}$, $\tau_{i}=t_{0}+i \Delta t, i \in \llbracket 0, N-1 \rrbracket$, and $\mathbf{r}_{i}=\mathbf{r}\left(\tau_{i}\right), \mathbf{r}_{0}=\mathbf{x}_{0}, \mathbf{r}_{N}=\mathbf{x}$, if the Hamiltonian $H$ is timeindependent, then $\frac{d H}{d t}=\frac{\partial H}{\partial t}=0$ and the equations (15) give the propagator which can be written as $a(\min ,+)$ path integral

$$
\begin{aligned}
S_{E L}\left(\mathbf{x}, t ; \mathbf{x}_{0}, t_{0}\right) & =\left\{\lim _{N \rightarrow+\infty} \bigotimes_{i=0}^{N-1}\left(\int_{\mathbf{r}(\tau), \tau_{i} \leq \tau \leq \tau_{i+1}}^{\oplus} d \mathbf{r}\right)\right\}\left(-\int_{t_{0}}^{t} H(\mathbf{x}, \nabla S ; s) d s\right) \\
& \equiv \int_{\mathbf{x}_{0}, t_{0}}^{\mathbf{x}, t} \mathcal{D}_{\oplus}[\mathbf{x}(\cdot)] \int_{t_{0}}^{t}-H(\mathbf{x}, \nabla S ; s) d s,
\end{aligned}
$$

where $\mathcal{D}_{\oplus}[\mathbf{x}(\cdot)]$ defines the $(\min ,+)$-action functional measure. 
This yields obviously to the fact that it exists a duality between the usual representation of a particle, and its action, with mean of the Euler-Lagrange action, which is the propagator of Hamilton-Jacobi equations (15).

\subsection{Semi-classical convergence}

Let us consider the semi-classical case where the evolution of the wave function $\psi(\mathbf{x}, t)$ of a massive particle $m$ verifies the Schrödinger equation

$$
\left\{\begin{array}{l}
i \hbar \frac{\partial}{\partial t} \psi(\mathbf{x}, t)=\left(\frac{-\hbar^{2}}{2 m} \Delta+V(\mathbf{x})\right) \psi(\mathbf{x}, t), \forall t>t_{0}, \forall \mathbf{x} \in \mathbb{R}^{3} \\
\psi\left(\mathbf{x}, t_{0}\right)=\psi_{0}(\mathbf{x}) .
\end{array}\right.
$$

With the variable change $\psi(\mathbf{x}, t)=\sqrt{\rho^{(\hbar)}(\mathbf{x}, t)} e^{i \frac{S^{(\hbar)}(\mathbf{x}, t)}{\hbar}}$, the Schrödinger equation can be splitted into real and imaginary parts, giving thus the so-called Madelung equations [36],

$$
\left\{\begin{array}{l}
\frac{\partial S^{(\hbar)}(\mathbf{x}, t)}{\partial t}+\frac{1}{2 m}\left(\nabla S^{(\hbar)}(\mathbf{x}, t)\right)^{2}+\left(V(\mathbf{x})-\frac{\hbar^{2}}{2 m} \frac{\Delta \sqrt{\rho^{(\hbar)}(\mathbf{x}, t)}}{\sqrt{\rho^{(\hbar)}(\mathbf{x}, t)}}\right)=0 \\
\frac{\partial \rho^{(\hbar)}(\mathbf{x}, t)}{\partial t}+\nabla \cdot\left(\rho^{(\hbar)}(\mathbf{x}, t) \frac{\nabla S^{(\hbar)}(\mathbf{x}, t)}{m}\right)=0 \\
\rho^{(\hbar)}\left(\mathbf{x}, t_{0}\right)=\rho_{0}^{(\hbar)}(\mathbf{x}) \text { and } S^{(\hbar)}\left(\mathbf{x}, t_{0}\right)=S_{0}^{(\hbar)}(\mathbf{x}) .
\end{array}\right.
$$

One considers below two cases depending on the preparation of the particles [20, 21].

Definition 12 (Semi-Classical undiscerned particle) - A quantum particle is said to be semiclassical undiscerned if its initial probability density $\rho_{0}^{(\hbar)}(\mathbf{x})$ and its initial action $S_{0}^{(\hbar)}(\mathbf{x})$ are regular functions $\rho_{0}(\mathbf{x})$ and $S_{0}(\mathbf{x})$ not depending on $\hbar$.

Example 7 It is the case of non-interacting particles set, all prepared in the same way : a free particle beam in a linear potential, an electronic or $C_{60}$ beam in the Young's slits diffraction, or an atomic beam in the Stern-Gerlach experiment.

Definition 13 (Semi-Classical discerned particle) - A quantum particle is said to be semiclassical discerned if its initial probability density $\rho_{0}^{\hbar}(\mathbf{x})$ converges, when $\hbar \rightarrow 0$, to a Dirac distribution and if its initial action $S_{0}^{\hbar}(\mathbf{x})$ is a regular function $S_{0}(\mathbf{x})$ not depending on $\hbar$.

Example 8 This situation occurs when the wave packet corresponds to a quasi-classical coherent state, introduced in 1926 by Schrödinger [41]. The field quantum theory and the second quantification are built on these coherent states [17]. The existence for the hydrogen atom of a localized wave packet whose motion is on the classical trajectory (an old dream of Schrödinger's) was predicted in 1994 by Bialynicki-Birula, Kalinski, Eberly, Buchleitner and Delande [3, 5, 6], and discovered recently by Maeda and Gallagher [37] on Rydberg atoms.

Theorem 3 For semi-classical undiscerned quantum particles, the probability density $\rho^{(\hbar)}(\mathbf{x}, t)$ and the action $S^{(\hbar)}(\mathbf{x}, t)$, solutions to the Madelung equations (28), converge when $\hbar \rightarrow 0$, to the classical density $\rho(\mathbf{x}, t)$ and the classical action $S_{H J}(\mathbf{x}, t)$, which are solutions of the statistical HamiltonJacobi equations (16). 
Proof 3 Let us write the wave function $\psi(\mathbf{x}, t)=\rho^{(\hbar)}(x, t) e^{\frac{i}{\hbar} S^{(\hbar)}(\mathbf{x}, t)}$ with mean of $\psi_{0}(\mathbf{x})$ using the Feynman path integral formalism[13, 42, 12]. Expression (24) becomes then

$$
\begin{aligned}
\psi(\mathbf{x}, t) & =\int d \mathbf{x}_{0} \cdot \psi_{0}\left(\mathbf{x}_{0}\right) \cdot \int_{\mathbf{x}_{0}, t_{0}}^{\mathbf{x}, t} \mathcal{D}[\mathbf{y}] \cdot e^{\frac{i}{\hbar} S_{E L}\left(\mathbf{y}, t \mid \mathbf{x}_{0}, t_{0}\right)}, \\
& =\int d \mathbf{x}_{0} \psi_{0}\left(\mathbf{x}_{0}\right) \cdot F_{(\hbar)}\left(t, t_{0}\right) \cdot e^{\frac{i}{\hbar} S_{E L}\left(\mathbf{x}, t ; \mathbf{x}_{0}, t_{0}\right)} \\
& \left.=\int d \mathbf{x}_{0} F_{(\hbar)}\left(t, t_{0}\right) \cdot \sqrt{\rho_{0}^{(\hbar)}\left(\mathbf{x}_{0}\right)} \cdot e^{\frac{i}{\hbar}\left\{S_{0}^{(\hbar)}\left(\mathbf{x}_{0}\right)+S_{E L}\left(\mathbf{x}, t ; \mathbf{x}_{0}, t_{0}\right)\right.}\right\}
\end{aligned}
$$

where $F_{(\hbar)}\left(t, t_{0}\right)$ is a function independent of $\mathbf{x}$ and $\mathbf{x}_{0}$ and $\hbar$-parameterized.

Since the quantum particle is undiscerned, the density and initial action are $\hbar$ independent and then

$$
\psi(\mathbf{x}, t)=\rho^{(\hbar)}(x, t) e^{\frac{i}{\hbar} S^{(\hbar)}(\mathbf{x}, t)}=\int d \mathbf{x}_{0} F_{(\hbar)}\left(t, t_{0}\right) \cdot \sqrt{\rho_{0}\left(\mathbf{x}_{0}\right)} \cdot e^{\frac{i}{\hbar}\left\{S_{0}\left(\mathbf{x}_{0}\right)+S_{E L}\left(\mathbf{x}, t ; \mathbf{x}_{0}, t_{0}\right)\right.} .
$$

When $\hbar \rightarrow 0$, the stationary phase approximation yields to[13, 14]

$$
S^{(\hbar)}(\mathbf{x}, t) \stackrel{\hbar \rightarrow 0}{\sim} \min _{\mathbf{x}_{0}}\left\{S_{0}\left(\mathbf{x}_{0}\right)+S_{E L}\left(\mathbf{x}, t ; \mathbf{x}_{0}, t_{0}\right)\right\}=S_{H J}(\mathbf{x}, t)
$$

That is to say that the quantum action $S^{(\hbar)}(\mathbf{x}, t)$ converges to the Hamilton-Jacobi action $S_{H J}(\mathbf{x}, t)$, already defined in (23), and solution of equations (16).

Moreover, since the quantum probability density $\rho^{(\hbar)}(\mathbf{x}, t)$ satisfies the continuity equation, and since $S^{(\hbar)}(\mathbf{x}, t)$ tends towards $S_{H J}(\mathbf{x}, t)$, one deduces that $\rho^{(\hbar)}(x, t)$ converges to the classical probability density $\rho(\mathbf{x}, t)$, which satisfies the continuity equation as well. One obtains thus both announced convergences[20].

Semi-classical undiscerned quantum particle is described by the Madelung equations (28) which converge to statistical Hamilton-Jacobi equations (16), corresponding to undiscerned classical particle. In this case, the density and the action are not sufficient to describe a classical particle. To know their positions at time $t$, it is necessary to know its initial positions $\mathbf{x}_{0}$. It is therefore logical to do the same in quantum mechanics. One considers this undiscerned quantum particle as a classical particle and concludes that a semi-classical undiscerned quantum particle is not completely described by its wave function. It is necessary to add its initial position and it becomes natural to introduce the de Broglie-Bohm interpretation [10, 4]. In this interpretation, the two first postulates of quantum mechanics, describing the quantum state and its evolution, must be completed. First, at initial time $t_{0}$, the state of the particle has to be given by the initial wave function $\psi\left(\mathbf{x}, t_{0}\right)=\psi_{0}(\mathbf{x})$ (a wave packet) and its initial position $\mathbf{r}\left(t_{0}\right)$; Second, the evolution of the wave function and the particle position are described respectively by the usual Schrödinger equation $(27)$ and $\frac{d}{d t} \mathbf{r}(t)=\left.\frac{1}{m} \nabla S^{(\hbar)}(\mathbf{x}, t)\right|_{\mathbf{x}=\mathbf{r}(t)}$.

\section{Conclusion}

One has shown in this article how some tools from tropical and idempotent mathematics, the socalled $(\mathrm{min},+)$-algebra and (min, + )-analysis, which were originally devoted to practical problems in operation research, graph theory, non-linear functional analysis, have permitted to develop a calculus framework, well-suited for the study and re-interpretation of Least Action Principle in classical mechanics. Moreover, many non-linear problems occurring in physics can be treated as linear within this $(\min ,+)$ framework.

Some elements of $(\mathrm{min},+)$-algebra and $(\min ,+)$-analysis have been introduced in order to exhibit 
some useful techniques for solving non-linear Partial Differential Equations (PDE) with mean of algebraic deformations, and to develop Distribution Theory and Functional Analysis based on $(\mathrm{min},+)$ integration, scalar product and Fourier-Legendre transform. Some applications to multi-resolution analysis of fractal and multi-fractal signals [32, 35], and complex variational calculus have already been explored in a previous paper[31].

The Bellman Theorem gives a very relevant approach to understand the underlying fractality structure guiding the Least Action Principle. The fact that the well-known shortest path research problem in a graph can be related at the continuous limit to this principle in classical mechanics, has permitted in a first step to use $(\mathrm{min},+)$ calculus to distinguish two kinds of actions, the Euler-Lagrange and the Hamilton-Jacobi one, depending on initial and limit conditions. In a second step, it has been shown that each action verifies different PDEs, and that the Hamilton-Jacobi action can be expressed as a $(\min ,+)$ path integral while Euler-Lagrange action is the propagator or the singular solution of the Hamilton-Jacobi equations. Moreover, this implies that it exists a duality between particle and its action.

An important result is that the semi-classical limit of an undiscerned quantum particle action converges to the Hamilton-Jacobi one, which is a relevant quantity in classical mechanics. One has used at the end of this paper those previous developments, to justify that the de Broglie-Bohm interpretation of quantum mechanics can keep coherent semi-classical convergences of undiscerned quantum particle action with the existence of trajectories and duality particle-action.

\section{Acknowledgements}

We thank Professors Lyazid Chetouani, Janos Polonyi and René Voltz for interesting and useful discussions about path integrals and least-action principles.

\section{References}

[1] Carré BA, An algebra for network routing problems. J. Inst. Maths Applics., 19, page 381, 1971.

[2] R. Bellman, Dynamic Programming. Princeton, Princeton University, 1957.

[3] Kalinski M., Eberly J. H., Bialynicki-Birula I., Lagrange equilibrium points in celestial mechanics and nonspreading wave packets for strongly driven rydberg electrons. Phys. Rev. Lett., pages 73, 1777, 1994.

[4] D. Bohm, A suggested interpretation of the quantum theory in terms of "hidden" variables. Phys. Rev., pages 85, 166-193, 1952.

[5] Delande D., Buchleitner A., Non-dispersive electronic wave packets in multiphoton processus. Phys. Rev. Lett., pages 75, 1487, 1995.

[6] Delande D., Zakrzewski J., Buchleitner A., Non-dispersive wave packets in periodically driven quantum systems. Physics Reports, pages 368, 409-547, 2002.

[7] Benzaken C., Structure algébrique des cheminements : pseudotreillis, gerbier de carré nul, in : Biorcu G. 5Ed. Networks and swatching theory, Academic Press, 1968.

[8] Demichev A. P., Chaichian M., Path Integrals in Physics Volume 1 : Stochastic Process \& Quantum Mechanics. Taylor \& Francis, 2001. 
[9] Hervé P. Cruon R., Quelques résultats relatifs à une structure algébrique et son application au problème central de l'ordonnancement. 1965.

[10] de Broglie L., La mécanique ondulatoire et la structure atomique de la matière et du rayonnement. Journal de Physique, pages 8, 225-241, 1927.

[11] L. Euler, Methodus Inveniendi Lineas Curvas Maximi Minive Proprietate Gaudentes. Bousquet, Lausanne et Geneva, 1744.

[12] R. Feynman and A. Hibbs, Quantum Mechanics and Path Integrals. McGraw-Hill, 1965.

[13] R. Feynman and A. Hibbs, Quantum Mechanics and Path Integrals. McGraw-Hill, pages 41-64, 1965.

[14] R. Feynman and A. Hibbs, Quantum Mechanics and Path Integrals. McGraw-Hill, pages 58-60, 1965.

[15] R. P. Feynman, Space-time approach to non-relativistic quantum mechanics. Reviews of Modern Physics, 20 (2):367-387, 1948.

[16] Litvinov G.L., The Maslov dequantization, idempotent and tropical mathematics : A brief introduction. Journal of Mathematical Sciences, v. 140, (3), 2007, pages 426-444, 2007.

[17] R. J. Glauber, Quantum Optics and Electronics, Les Houches Lectures 1964, C. deWitt, A. Blandin and C. Cohen-Tanoudji. Gordon and Breach, New York, 1965.

[18] M. Gondran, Analyse minplus. C. R. Acad. Sci. Paris, 323:371-375, 1996.

[19] M. Gondran, Convergences de fonctions à valeurs dans $\mathbb{R}^{k}$ et analyse minplus complexe. C. $R$. Acad. Sci. Paris, 329:783-788, 1999.

[20] Gondran A., Gondran M., Discerned and non-discerned particles in classical mechanics and convergence of quantum mechanics to classical mechanics. Annales de la Fondation Louis de Broglie, pages 39, 117-135, 2011.

[21] Gondran A., Gondran M., The two limits of the schrödinger equation in the semi-classical approximation : discerned and non-discerned particles in classical mechanics. Foundations of Probability and Physics-6, AIP Conf. Proc. 1424,111-115 (2012)., pages 1424, 111-115, 2012.

[22] Tomescu I., Sur les méthodes matricielles dans la théorie des réseaux. 1966.

[23] Kutzman J., Théorie des réseaux graphes. Dunod-Paris, 1972.

[24] J.L. Lagrange, Mécanique Analytique. Gauthier-Villars, Paris, translated by V. Vagliente and A. Boissonade (Klumer Academic, Dordrecht, 2001), 2nd edition, 1888.

[25] Gondran M., Problèmes combinatoires et programmation en nombres entiers. Thèse de Doctorat d'Etat. University of Paris VI, 1974.

[26] Gondran M., L'algorithme glouton dans les algèbres de chemin. Bulletin des Etudes et de la Recherche, EDF, Série C, pages 1, 25-32, 1975.

[27] Gondran M., Path algebra and algorithms. B. Roy (Ed.), Combinatorial Programming : Methods and Applications, Reidel, Dordrecht, pages 137-148, 1975. 
[28] Gondran M., Valeurs propres et vecteurs propres en classification hiérarchique. RAIROTheoretical Informatics and Applications, pages 10 (1) 39-46, 1976.

[29] Gondran M., Les éléments p-réguliers dans les dioïdes. Discrete Mathematics, pages 25 : 33-39, 1979.

[30] Gondran M., Valeurs propres et vecteurs propres en analyse des préférences. Note EDF, pages HI-3199, 1979.

[31] A. Gondran, M. Gondran, A. Kenoufi, Complex variational calculus with mean of (min, +)analysis. Trends in Applied and Computational Mathematics, 18(3):1-19, 2017.

[32] A. Kenoufi, M. Gondran, Numerical calculations of Hölder exponents for the Weierstrass functions with (min,+)-wavelets. Trends in Applied and Computational Mathematics, 15(3):261273.

[33] M. Minoux, M. Gondran, Graphs and Algorithms. Wiley-Interscience, 1984.

[34] M. Minoux, M. Gondran, Graphs, Dioids and Semirings. Springer, 2008.

[35] T. Lehner, M. Gondran, A. Kenoufi, Multi-fractal analysis for Riemann Serie and Mandelbrot Binomial Measure with (min, +)-wavelets. Trends in Applied and Computational Mathematics, 17(2):247-263, 2016.

[36] E. Madelung, Quantentheorie in hydrodynamischer form. Zeit. Phys., pages 40, 322-6, 1926.

[37] Gallagher T.F., Maeda H., Non dispersing wave packets. Phys. Rev. Lett., pages 92, 133004-1, 2004.

[38] V. Maslov, Operational calculus. Mir, Moscow, 1987.

[39] P. Del Moral, A survey of Maslov optimization theory. V. N. Kolokoltsov and V. P. Maslov, Idempotent Analysis and Applications, Kluwer Acad. Publ., Dordrecht, 1997.

[40] Ferland J., Robert P, Généralisation de l'algorithme de warshall. 1968.

[41] E. Schrödinger, Der stetige bergang von der mikro-zur makromechanik. Naturwissenschaften, pages 14, 664-666, 1926.

[42] L.S. Schulmann, Techniques and Applications of Path Integration. John Wiley \& Sons, New York, 1981.

[43] Peteanu V., An algebra of the optimal path in networks. 1967.

[44] S.N. Samborski, V. Maslov, Idempotent analysis. Advances in Soviet Mathematics, American Mathematical Society, 13, 1992.

[45] J. H. Van Vleck, The correspondence principle in the statistical interpretation of quantum mechanics. Proceedings of the National Academy of Sciences of the United States of America, 14 (2):178-188, 1928.

[46] V.N. Kolokoltsov, V.P. Maslov, Idempotent analysis and its applications in optimal control. Nauka, 1994. 